\title{
Hepatitis C in the United States
}

\author{
Scott D. Holmberg, M.D., M.P.H., Philip R. Spradling, M.D., Anne C. Moorman, M.P.H., and \\ Maxine M. Denniston, M.S.P.H. \\ Epidemiology and Surveillance Branch, Division of Viral Hepatitis, Centers for Disease Control \\ and Prevention, Atlanta
}

\begin{abstract}
Care for hepatitis $\mathrm{C}$ is evolving rapidly, with increasingly effective and better-tolerated antiviral therapies being evaluated and approved for use. It's clear, however, that not everyone who would qualify for therapy has been tested and identified, referred for appropriate care, and offered or given the best therapy available. Furthermore, currently used antiviral drugs - pegylated interferon and ribavirin "base" plus either telaprevir or boceprevir - can cost more than $\$ 70,000$ for a full course of therapy. It is expected that the new oral antiviral agents will be just as expensive, at least in the short term. All these factors affect personal, medical, public health, and national policy decisions. One fundamental problem in making such decisions is that it's difficult to estimate the number of people with chronic hepatitis $\mathrm{C}$ virus (HCV) infection in the United States who have been identified and have received appropriate care.
\end{abstract}

Over the past 4 years, members of the Division of Viral Hepatitis at the Centers for Disease Control and Prevention (CDC) have executed and analyzed two large studies of hepatitis C in the United States. Researchers conducting the Chronic Hepatitis Cohort Study (CHeCS) are currently examining records from more than 13,000 patients with hepatitis $\mathrm{C}$ (and more than 3500 with hepatitis B) who have been seen at four health care organizations in the United States (in Detroit, Michigan; Danville, Pennsylvania; Portland, Oregon; and Honolulu, Hawaii) since 2006. These patients are drawn from a population of about 1.6 million adults who have received care at these four sites during the approximately 6 years for which retrospective and prospective analysis has been under way. ${ }^{1,2}$ The National Health and Nutrition Examination Survey (NHANES) takes a different approach: random sampling of approximately 5000 non-institutionalized U.S. civilians per year, using standardized household interviews, physical examinations, and testing of serum samples. ${ }^{3}$ Details and results of these two studies give a consistent picture of the status of HCV infection in the United States (see flow chart).

An examination of the prevalence of chronic HCV infection in the United States during the period 1999 through 2002, based on NHANES data and factoring in persons who were institutionalized, incarcerated, or homeless, suggested that there were about 3.5 million HCV-infected U.S. residents. ${ }^{4}$ According to an as-yet-unpublished study by Denniston et al.,

The findings and conclusions in this article are those of the authors and do not necessarily represent the views of the Centers for Disease Control and Prevention.

Disclosure forms provided by the authors are available with the full text of this article at NEJM.org. 
a more recent prevalence estimate based on NHANES data from 2003 through 2010 reveals the effect of increasing mortality on this population. These analyses indicate that a reasonable estimate of the current number of infected people in the United States is about 3.2 million.

The CHeCS investigators examined 1.2 million people who used the four integrated medical care systems during 2006 through 2008, and 57\% of the number estimated to have HCV infection had actually been tested and identified as infected. In the broader population from which the 30,140 NHANES participants were drawn, 50\% of persons who had tested positive for antibodies to $\mathrm{HCV}$ and provided information during in-depth telephone interviews were aware of their $\mathrm{HCV}$-infection status before being notified of that infection by the NHANES. ${ }^{3}$ The CHeCS researchers are currently examining reasons why the people who were found to be infected in their study had or had not been tested previously. In the population on which the CHeCS draws, less than half of people who had had two or more abnormal alanine aminotransferase results were subsequently tested for HCV infection. ${ }^{2}$

As an indication of access to care, of the first $8810 \mathrm{CHeCS}$ patients - who are receiving care at integrated health care organizations $-62 \%$ had private insurance, $35 \%$ had public insurance (Medicare or Medicaid), and 3\% had none. ${ }^{1}$ In the NHANES, 128 of $170 \mathrm{HCV}$ infected people who responded to follow-up surveys $(75 \%)$ said they had health insurance, ${ }^{3}$ but the type of insurance was not included in the analysis.

As for follow-up care, of 9086 adults in the population from which the CHeCS cohort was drawn who had a positive HCV-antibody test during 2006 through 2008, a total of 3428 (38\%) had no follow-up HCV RNA testing documented in the electronic database ${ }^{1}$; but since there was laboratory evidence of HCV RNA testing for 63\% (though results of tests performed outside the participating health care networks could not be obtained), this percentage should be viewed as the minimum proportion who received at least some followup care. In the NHANES, 77\% of respondents indicated that they had seen a clinician after their first HCV test result; these included 71 of 82 persons who knew they were infected before they were tested in the NHANES (87\%) and 59 of 85 persons who discovered their infection because of their participation (69\%). From these data it seems reasonable to deduce that 63 to $77 \%$ of people who have tested positive for HCV antibodies - 32 to $38 \%$ of all HCV-infected people in the United States - received follow-up hepatitis care.

Among those receiving care, such as the 8810 who were initially examined in the $\mathrm{CHeCS}$, 5540 (63\%) had had at least one HCV RNA measurement between 2001 and 2010. Of the $\mathrm{HCV}$-infected people in the CHeCS - people who are more likely than average to be receiving specialist care for $\mathrm{HCV}-3380(38 \%)$ had undergone a liver biopsy between 2001 and 2010. ${ }^{1}$ In the NHANES, of 66 persons who said they received care for their HCV infection, 31 (47\%) said they had undergone a biopsy. These proportions translate to about 12 to $18 \%$ of the total $\mathrm{HCV}$-infected population.

In the CHeCS, $36 \%$ of people who knew they were infected - about $18 \%$ of the estimated total infected population who had been identified as infected - had evidence in their electronic or hardcopy chart of any treatment for HCV. ${ }^{1}$ In the NHANES, 22 of the 170 
HCV-infected persons who answered follow-up surveys (13\%) said they had received treatment for $\mathrm{HCV}$ infection. ${ }^{3}$

It is more difficult to determine whether treatment has been successful, but in the CHeCS the most recent test results indicated that HCV RNA was "undetectable" in $21 \%$ of patients, and $80 \%$ of patients with such results had documentation of having received antiviral therapy 1 - that is, about $17 \%$ of the total $\mathrm{CHeCS}$ cohort, or about 5 to $6 \%$ of all $\mathrm{HCV}$-infected people.

One limitation of both the CHeCS and the NHANES results is that because estimates are unavoidably based on progressively smaller numbers of patients, they have wide confidence intervals. Both studies were biased toward following (in the $\mathrm{CHeCS}$ ) or recruiting for interview (in the NHANES) persons who were more likely to have health insurance and to be receiving health care; thus, the resulting estimates may actually be high. Still, these data and estimates derive from two large U.S. studies using different methods and sampling sources, one a managed-care population (CHeCS) with the largest cohort of HCV-infected patients in the United States and the other the noninstitutionalized civilian U.S. population (NHANES). Despite the different methods and unavoidable selection biases, the results appear to be consistent and credible.

This big picture suggests that there are many points of intervention - or opportunities - to improve the identification and care of patients with $\mathrm{HCV}$ and to mitigate the increase in hospitalizations and deaths resulting from $\mathrm{HCV}$ infection. For example, the $\mathrm{CDC}$ recently recommended a one-time test for everyone born between 1945 and $1965^{5}$ to help identify the many infected people who would not be targeted for testing as the result of established riskbased testing strategies. Clearly, there is also a need to do a better job of getting HCVinfected persons who know their HCV status into care, evaluated, and, as appropriate, treated. It is past time to address more vigorously what Assistant Secretary for Health Howard Koh has called the silent epidemic of viral hepatitis.

\section{References}

1. Moorman AC, Gordon SC, Rupp LB, et al. Baseline characteristics and mortality among people in care for chronic viral hepatitis: the Chronic Hepatitis Cohort Study. Clin Infect Dis. 2013; 56:40-50. [PubMed: 22990852]

2. Spradling PR, Rupp L, Moorman AC, et al. Hepatitis B and C virus infection among 1. 2 million persons with access to care: factors associated with testing and infection prevalence. Clin Infect Dis. 2012; 55:1047-55. [PubMed: 22875876]

3. Denniston MM, Klevens RM, McQuillan GM, Jiles RB. Awareness of infection, knowledge of hepatitis $\mathrm{C}$, and medical follow-up among individuals testing positive for hepatitis C: National Health and Nutrition Examination Survey 2001-2008. Hepatology. 2012; 55:1652-61. [PubMed: 22213025]

4. Armstrong GL, Wasley A, Simard EP, Mc-Quillan GM, Kuhnert WL, Alter MJ. The prevalence of hepatitis C virus infection in the United States, 1999 through 2002. Ann Intern Med. 2006; 144:705-14. [PubMed: 16702586]

5. Smith BD, Morgan RL, Beckett GA, et al. Recommendations for the identification of chronic hepatitis C virus infection among persons born during 1945-1965. MMWR Recomm Rep. 2012; 61(RR-4):1-32. [Erratum, MMWR Recomm Rep 2012;61:886.]. 


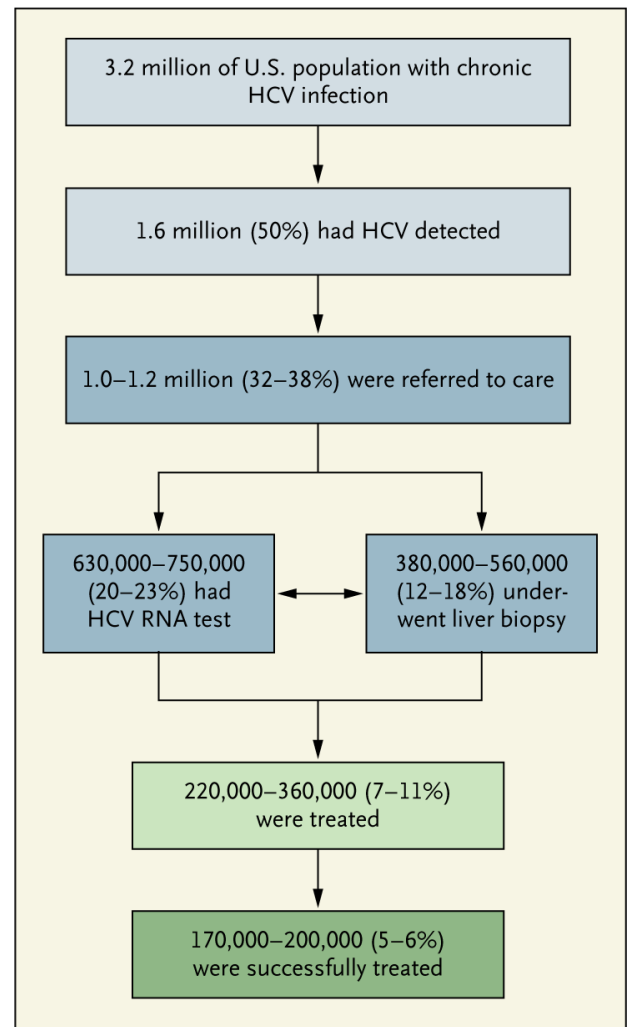

Figure 1. Hepatitis C Virus (HCV)-Infected Persons in the United States and Estimated Rates of Detection, Referral to Care, and Treatment

Percentages in parentheses are percentages of the total estimated HCV-infected population (3.2 million persons). 